\title{
Standardization of logic for a constant warning time control at automatic level crossings
}

\author{
M. Teramoto ${ }^{1}$, N. Miyaguchi ${ }^{1}$, K. Kumasaka ${ }^{2}$, R. Ishima ${ }^{3}$ \\ \& Y. Fukuta ${ }^{1}$ \\ ${ }^{1}$ East Japan Railway Company, Japan \\ ${ }^{2}$ Daido Signal Ltd., Japan \\ ${ }^{3}$ Japan International Consultants for Transportation Co., Ltd., Japan
}

\begin{abstract}
In Japan, there are a lot of high-density railway lines and along the line the long warning time at level crossings has become a social problem. Particularly, about 600 level crossings have a warning time of more than 40 minutes per hour in rush hour. As a result, the traffic of cars and pedestrians is disturbed for a long time. Eliminating level crossings by making overhead crossings has been attempted, but a lot of time and costs are necessary. Therefore, it is an urgent problem to shorten the warning time for such level crossings.

Although a constant warning time control for level crossings has been widely adopted at JR East as a countermeasure against the length of time that the level crossing barrier is closed, it requires a lot of hard work on its design and construction as well as testing because its control logic differs in level crossings. In order to cope with this situation, we employed a new train counting sequence, employing two types of counting logic to replace the track circuits. To realize this logic, we only need two starting points and one stopping point. When a train enters one of the two starting points, the train is counted according to the corresponding type. When the train leaves the stopping point, it is subtracted. Thus, we have achieved a train recognition sequence without track circuits. We can apply this simple sequence to all types of automatic level crossings. We are going to incorporate this logic in PLC (programmable logic controller) as a new microelectronic level crossing controller. It is expected to become a product after passing the field test. By using our development, it is expected to reduce the time for design, construction and testing. Furthermore, it is also expected to reduce the number of relays, which leads to a reduction in cost. By the above-mentioned
\end{abstract}


effect, our development leads to the promotion of the introduction of Constant Warning Time Control for Level Crossing and becomes a contribution to society. Keywords: level crossings, Constant Warning Time Control, level crossing controller, extraordinary long time closed level crossing, ATS-P.

\section{Introduction}

In Japan, there are a lot of barrier protected level crossings [1] and a lot of high-density railway lines. Therefore along the line, the long warning time of level crossings has become a social problem. Particularly, about 600 level crossings have a warning of more than 40 minutes per hour in rush hour. As a result, the traffic of cars and pedestrians is disturbed for a long time [2].

It has been tried to eliminate level crossings by making overhead crossings, but much time and cost are necessary. Therefore, it is an urgent problem to shorten the warning time for such level crossings.

A constant warning time control for level crossings has been widely adopted at JR East as a countermeasure against the length of time that a level crossing barrier is closed. Its control logic is realized by changing the starting point according to train types. It was introduced in 66 places by 2011 at automatic level crossings, and the effect of shortening warning time that we expected has been realised.

We will continue to introduce it in the future, but trouble with introduction is expected because we still need time for design, construction and testing.

This is because the constant warning time control logic differs in level crossings, even where there are relatively simple track layouts [3]. This is because train position detection methods are different from setting position patterns at the starting point, stopping point and track circuit. Therefore, our aim is to build a simple logic that can be applied to all automatic level crossings between stations $[4,5]$.

\section{Schematics of constant warning time control for level crossing}

\subsection{Constant warning time control system for automatic level crossing}

The warning time of level crossings is designed for the fastest train. Therefore, the warning time for level crossings increases when a low speed train stops at the station. Therefore, we have to identify the train type whether it is a high speed train passing through the station or a low speed train stopping. We usually use the train identification function of ATS-P, which is an ATP (Automatic Train Protection) system. The system configuration of constant warning time control for level crossings is shown in Figure 1. A typical system consists of the starting point for a passing train (detector A), the starting point for a stopping train (detector E), the stopping point (detector B), ATS-P and the level crossing controller. The level crossing controller consists of a microelectronic level crossing controller and a relay connections circuit. 
When a train approaches a level crossing, the ATS-P generates a brake pattern before the level crossing. The level crossing controller obtains train type information from the ATS-P. A passing train starts warning from detector A and a stopping train starts warning from detector E. Therefore, trains that stop can avoid an unnecessarily long warning time. In a case in which a stopping train maintains speed and passes the station by mistake, the ATS-P brake pattern can stop the train just before the level crossing. When the level crossing controller outputs information that a crossing gate is closed, the ATS-P eliminates the brake pattern.

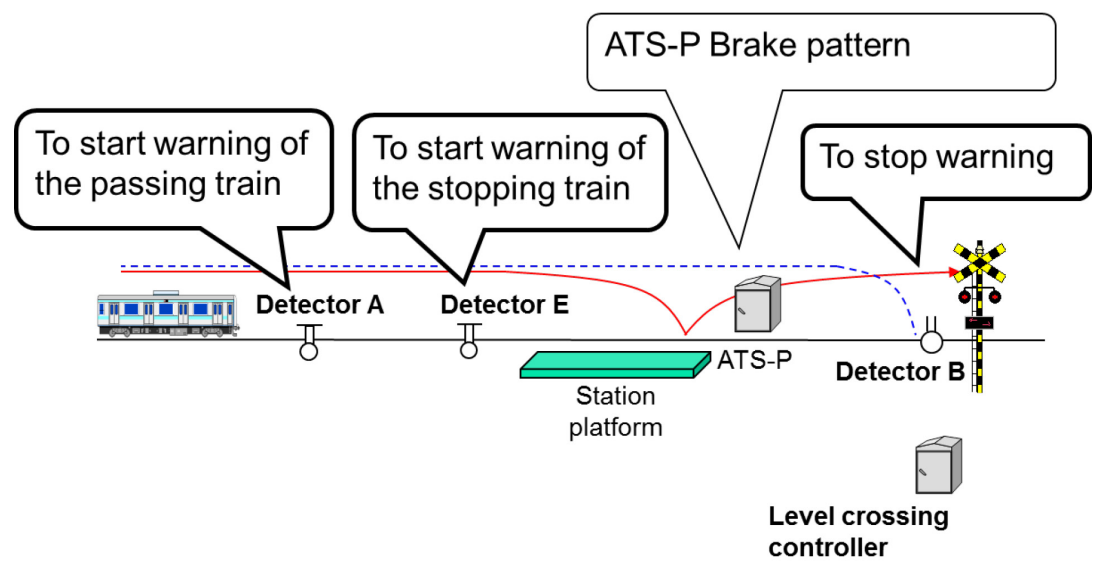

Figure 1: System configuration of constant warning time control of level crossing.

\subsection{Control logic by using relay connections}

Figure 2 shows the image of control logic by using relay connections. Typically, when we install a constant warning time control system by using relay connections, it has to be customized for each level crossing. Therefore, we still need to time for design, construction and testing.
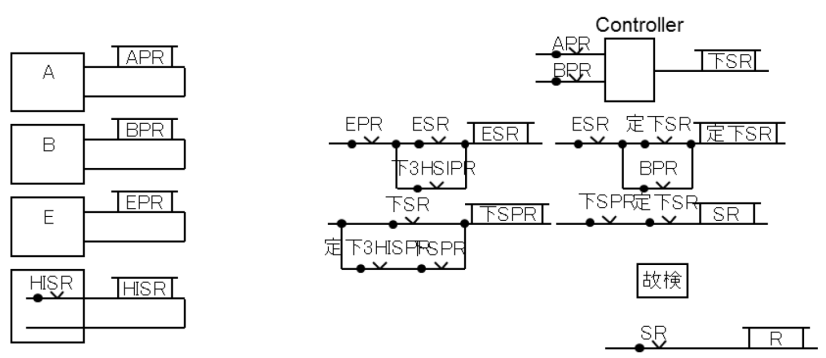

Figure 2: The image of control logic by using relay connections. 
In order to reveal the reason why we have to make a different design for each level crossing, we have investigated 66 actually operating level crossings that consist of track circuits and relay logics.

According to the investigation of the control tables and the connection diagrams of the 66 level crossings, track circuits have important roles to conventional control logic.

Figure 3 shows the positional relations between starting points (detector A, E) and track circuits. Figure 4 shows two points (detector E, B) ahead and track circuits. At least more than $3 \times 3$ setting position patterns appear because these patterns are combined. The result of the investigation about the relationship between each setting position pattern and the number of the roadway of level crossing is shown in Tables 1 and 2. In preparation for the next train closest to the first train that starts the warning, we have to employ a lot of warning logic. The logic is different because the method to detect the next train varies according to each setting position pattern.

Furthermore, the required number of track circuits to detect the next train is also different between each level crossing. Therefore, we must arrange track circuits one by one, and this results in the complexity of the warning logic.

Pattern A: Detectors $A$ and $E$ are installed in the same block.

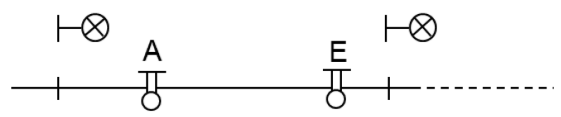

Pattern B: Detectors A and E are installed in the continuous blocks.

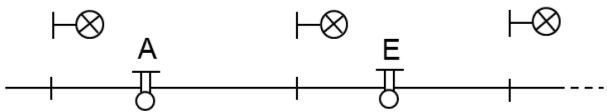
Pattern C: Detector E is installed in the block which is separated one or more blocks away from detector $\mathrm{A}$.

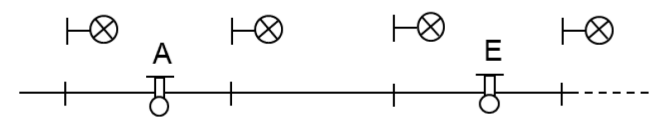

Figure 3: The positional relations between starting points (detector A, E) and track circuits. 
Pattern 1: Detectors $E$ and $B$ are installed in the same block.

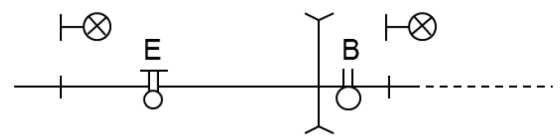

Pattern 2: Detectors $E$ and $B$ are installed in the continuous blocks.

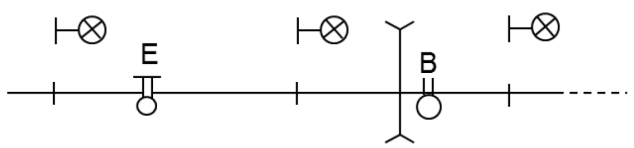

Pattern 3: Detector E is installed in the block which is separated one or more blocks away from detector $\mathrm{B}$

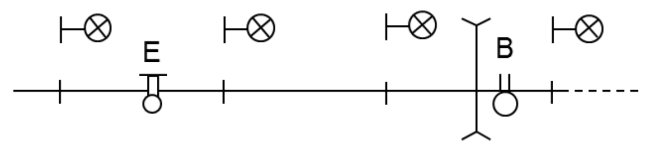

Figure 4: The positional relations between two points (the detectors $\mathrm{E}$ and B) ahead and track circuits.

Table 1: $\quad$ The relationship between setting position patterns (detectors $\mathrm{A}$ and $\mathrm{E}$, and the track circuit) and the number of level crossings.

\begin{tabular}{|c|c|}
\hline $\begin{array}{c}\text { The relationship between setting position patterns } \\
\text { (detectors A and E, and the track circuit) }\end{array}$ & $\begin{array}{c}\text { The number of } \\
\text { level crossings }\end{array}$ \\
\hline \hline Pattern A & 15 \\
\hline Pattern B & 49 \\
\hline Pattern C & 2 \\
\hline total & 66 \\
\hline
\end{tabular}

Table 2: $\quad$ The relationship between setting position patterns (the detectors $\mathrm{E}$ and $\mathrm{B}$, and the track circuit) and the number of level crossings.

\begin{tabular}{|c|c|}
\hline $\begin{array}{c}\text { The relationship between setting position patterns } \\
\text { (the detectors } \mathrm{E} \text { and } \mathrm{B} \text {, and the track circuit) }\end{array}$ & $\begin{array}{c}\text { The number of } \\
\text { level crossings }\end{array}$ \\
\hline \hline Pattern 1 & 2 \\
\hline Pattern 2 & 33 \\
\hline Pattern 3 & 31 \\
\hline total & 66 \\
\hline
\end{tabular}

In the next step, we investigated the way track circuits are used. We found that the track circuits are used for the following two purposes.

(a) Countermeasures against the incorrect barrier opening by the preceding train. 
The countermeasures against the incorrect barrier opening by the preceding train are realised by using relay connection, which is shown in Figure 5. In the case that the following train is between detectors $\mathrm{E}$ and $\mathrm{B}$, relay logic does not detect the position of the following train. Then the level crossing controller stops the warning and opens the barriers when the preceding train leaves detector B. To deal with this situation, the level crossing controller judges when to stop the warning and to open the barriers after checking the position of the following train by using the track circuit information.

(b) Countermeasures against the incorrect cancellation of the brake pattern by the following train.

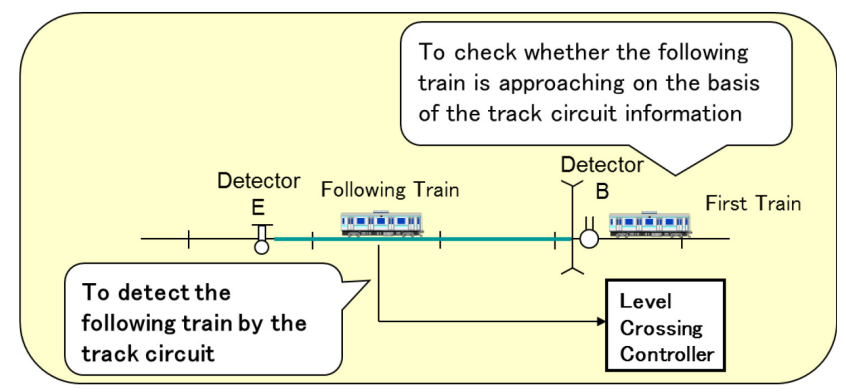

Figure 5: The countermeasures against the incorrect barrier opening by the preceding train.

Figure 6 shows a case where the brake pattern for the first stopping train is cancelled incorrectly when the following passing train reaches detector A. To deal with this situation, the conventional system checks the position of the preceding train by using the track circuit information. It is only when the preceding train has left that it cancels the preceding train's brake pattern to stop in the rear of the level crossing without a time delay.

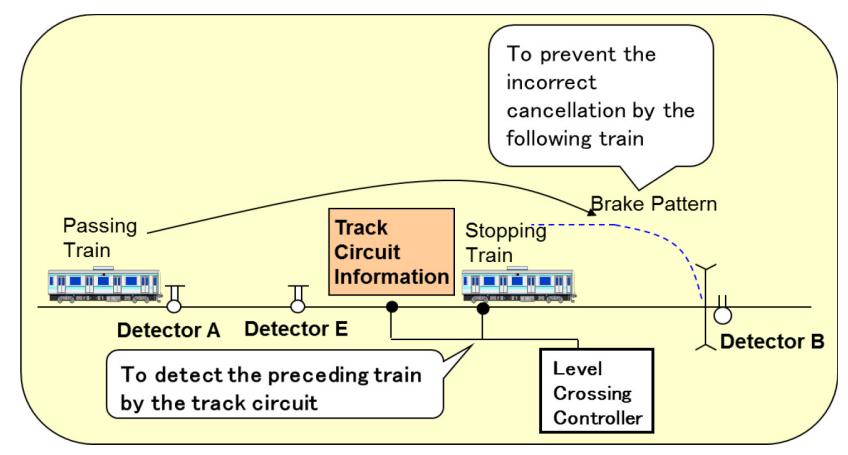

Figure 6: The countermeasures against the incorrect cancellation of the brake pattern by the following train. 


\section{Standardization of the logic of a constant warning time control at automatic level crossings}

\subsection{Contents of development}

Based on the above-mentioned analysis, we aim to reduce relay connections and standardize design by making software logic. Figure 7 shows the image of the reduction of the number of relays by using software. It shows that the software succeeds in reducing the number of relays.

Furthermore, the logic is expected to apply to every type of level crossing which is located between stations.

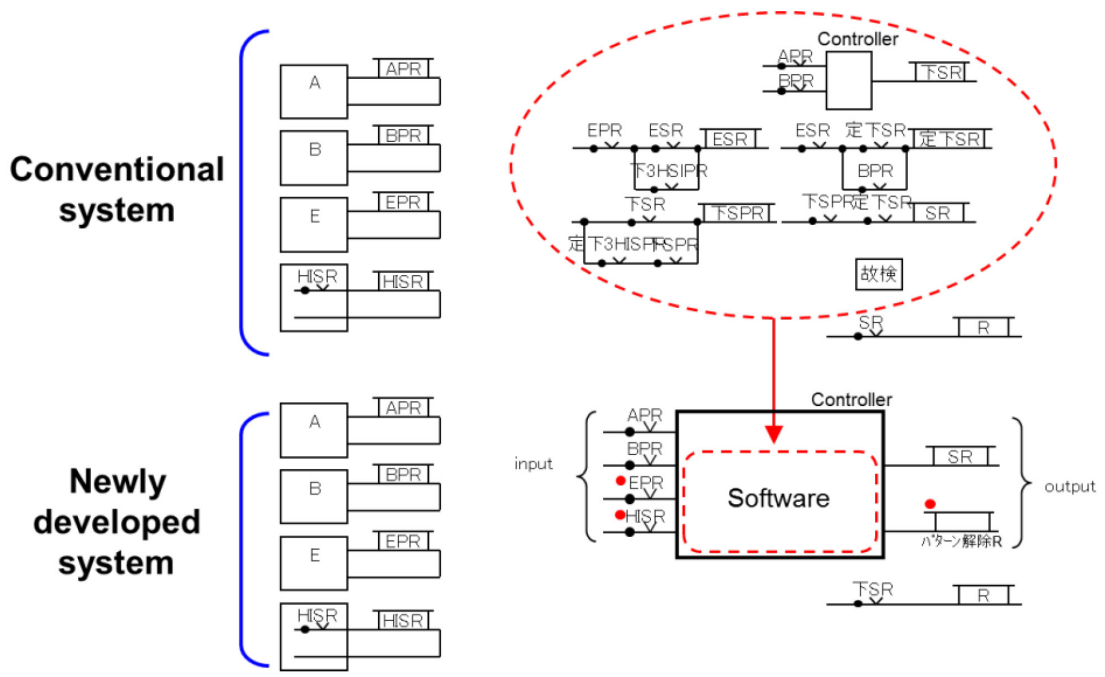

Figure 7: The image of reduction of the number of relays by using software.

\subsection{Standardization of logic}

By investigation of the conventional system, we have found that conventional relay logic entirely depends on the track circuits in order to achieve the two kinds of countermeasures discussed in the previous section, i.e. countermeasures against the incorrect barrier being opened by the preceding train and against the incorrect cancellation of the brake pattern by the following train. However, the positional relationships of the detectors and track circuits are too many to standardize. Therefore we have decided to construct the control logic without track circuits. If realized, many kinds of setting position patterns can be standardized to one setting pattern (Figure 8).

Since the order of the detectors is uniform, we can achieve standardized control logic. 
Using track circuits for logic

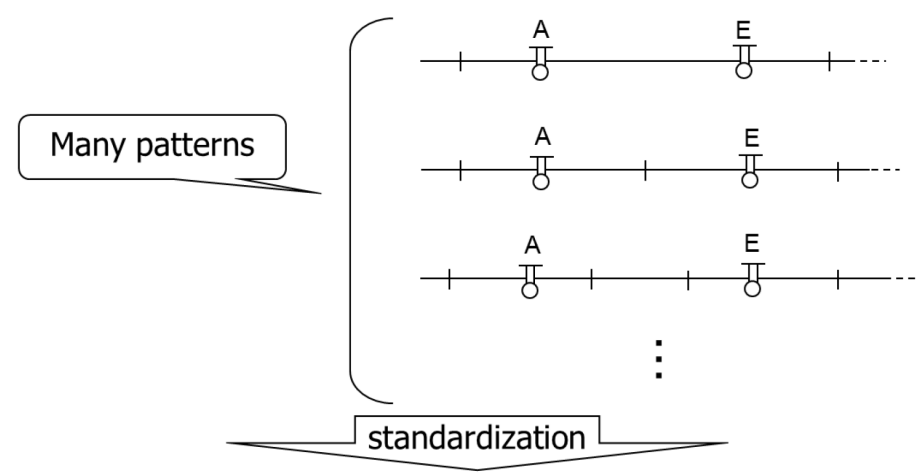

Not using track circuit for logic
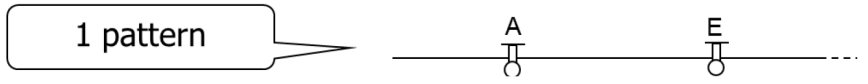

Figure 8: Standardization of position patterns.

We have utilized an already-existing microelectronic level crossing controller [6], and its control logic consists of three parts: (i) train count logic; (ii) warning control logic; (iii) diagnostic logic.

The number of trains running in the level crossing control area is counted by Train Count Logic (TCL). A train is counted after it crosses the starting point, and is subtracted after passing the stopping point. We have decided to extend the function of the train count, and Figure 9 explains the function of our logic. TCL counts the numbers of trains between detectors $\mathrm{A}$ and $\mathrm{B}$ (the $\mathrm{AB}$ counter calculates the number) and between detectors $\mathrm{E}$ detector $\mathrm{B}$ (the EB counter). In addition, the control logic judges whether the approaching train is the first train to arrive or not, and the starting point of the warning is adjusted according to the result.

In this case, the track circuit information for countermeasures against the incorrect barrier opening by the preceding train is unnecessary because the logic controller can manage the position of the following train by using TCL.

The "Diagnostic Logic" observes external situations such as detectors A and $\mathrm{B}$, and judges whether the level crossing system has failed or not.

Two types of countermeasures without using track circuits are as follows:

(a) The countermeasures against the incorrect barrier opening by the preceding train.

Our logic uses the two train counters to control the warning. When the value of the $\mathrm{AB}$ counter is zero and that of EB is also zero, our logic stops the warning and opens the barriers. If one or both of counters $A B$ and $E B$ is not zero, the logic continues the warning, as shown in Figure 10.

(b) The countermeasures against the incorrect cancellation of the brake pattern by the following train. 


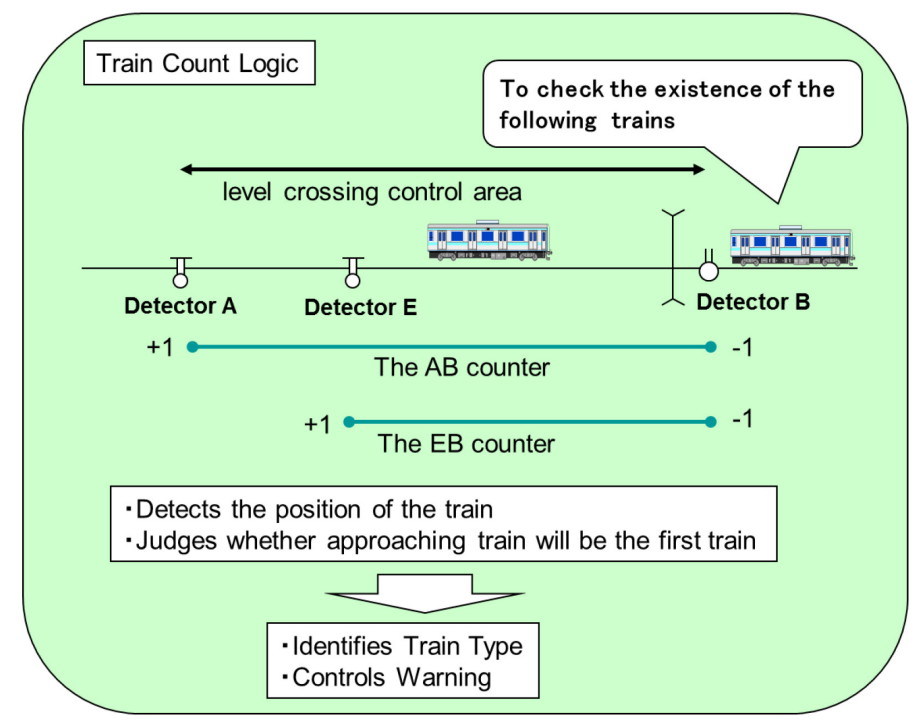

Figure 9: The feature of our development logic.

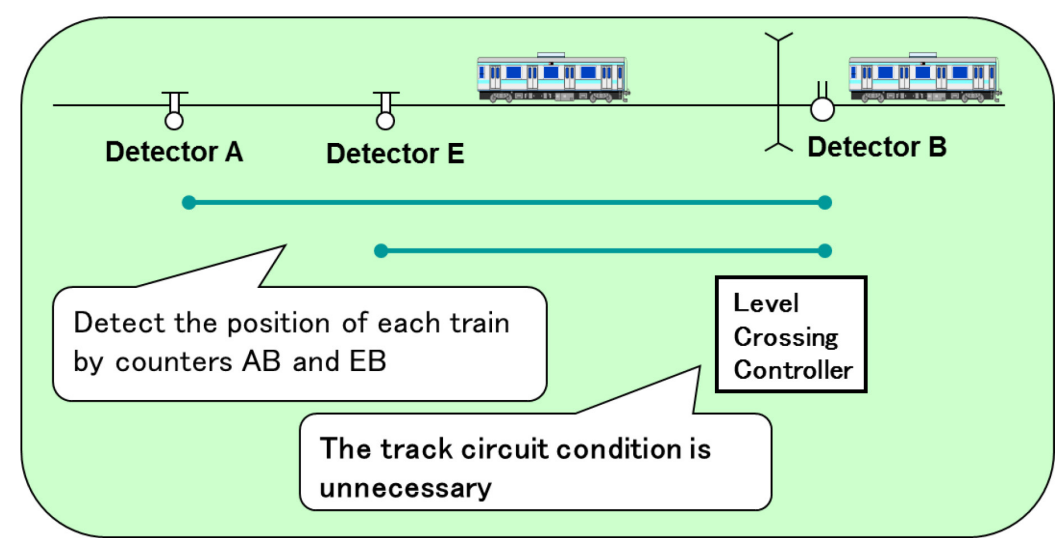

Figure 10: The countermeasures against the incorrect barrier opening by the preceding train without using the track circuits.

The reason why a countermeasure against the incorrect cancellation of the brake pattern by the following train is needed is that conventional level crossing control logic cannot grasp the order of trains. Thus, if we do not consider countermeasures, the brake pattern applied to the first stopping train could be cancelled and the level crossing warning incorrectly commences.

We constructed a new control logic as follows. Firstly, the logic judges whether a train is the first train. Secondly, only the train type of the first train is identified and the level crossing warning commences. 
For the following train, the logic only detects the position and does not control warning commencement. It stops the warning only after all trains have left the level crossing control section.

By using this logic we succeeded in achieving countermeasures against incorrect cancellation of brake patterns by the following train without using track circuit information (Figure 11).

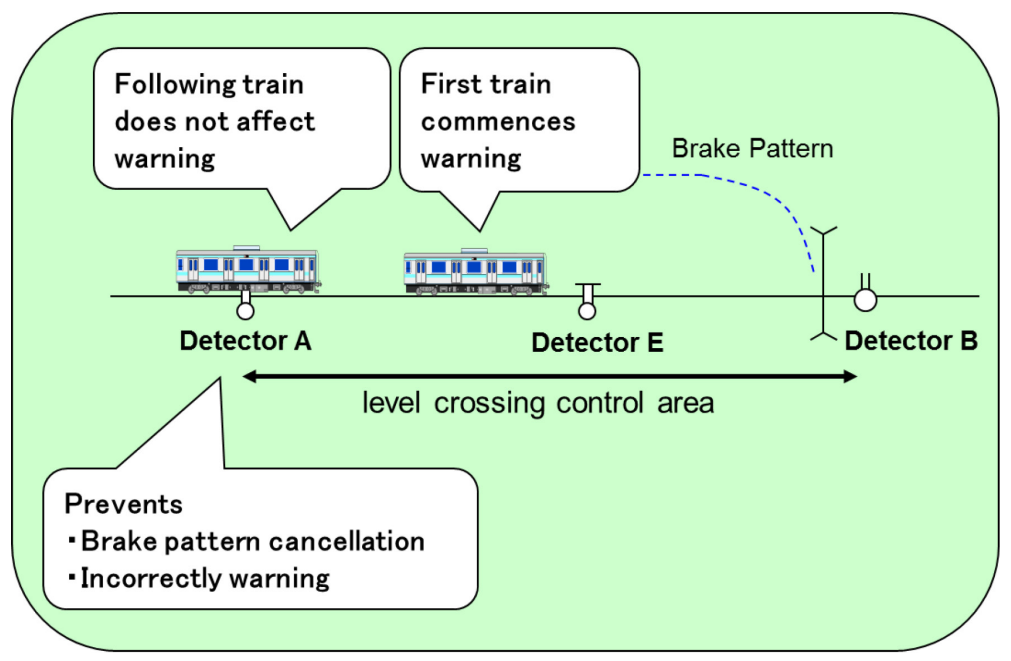

Figure 11: The countermeasures against the incorrect cancellation of the brake pattern by the following train without using track circuit information.

As discussed above, we have succeeded in developing two kinds of countermeasures and constructing a simple logic that does not require a track circuit.

\section{Project plan}

This new microelectronic level crossing controller with its built-in logic is currently in the final stage of product development.

We are going to incorporate this logic in PLC (programmable logic controller) as the new microelectronic level crossing controller. We are going to conduct field tests for the purpose of evaluating functions and environmental resistance during long-time operation in the field environment. We are planning to carry out the field test at Hikawa crossing between Omiya and Toro on the Utsunomiya line, from July 2014 to February 2015. It is expected to become a product after passing the field test.

By using our development, it is expected that the time for design, construction and testing will be reduced. Furthermore, it is also expected that the number of relays will be reduced, which will lead to a reduction in cost. 
By the above-mentioned effect, our development leads to the promotion of the introduction of Constant Warning Time Control for Level Crossings and will become a contribution to society.

\section{Conclusion}

In Japan the extraordinary long time of the warning at level crossings is a social problem. To solve this problem effectively we have developed logic for constant warning time control at automatic level crossings. If we used conventional logic, a lot of hard work on its design and construction as well as testing would be required as the logic is different at each crossing. We investigated the conventional system and revealed the reason. This is because the current logic relies on track circuits for countermeasures, i.e. countermeasures against the incorrect barrier opening by the preceding train and against the incorrect cancellation of the brake pattern by the following train. This leads to many kinds of logic patterns.

We have taken advantage of the train counter function; in particular, we have employed two train counters, which make it possible to construct logic without track circuit information and to standardize the logic through software development. We will be able to commonly apply our logic to all automatic level crossings between stations. We succeeded in operating PLC with this software, a controller which works as a new microelectronic level crossing controller. Thus, we can easily design and construct as well as conduct tests. Furthermore, we will be able to reduce the number of relays through software implementation. By the above-mentioned effect, our development leads to the promotion of the introduction of a Constant Warning Time Control for Level Crossing and will become a contribution to society.

\section{References}

[1] International Barriers to Level Crossing, Written and edited by Ian Mc Cullough, IRSE-ITC on behalf of the International Technical Committee of the IRSE. http://www.irse.org/knowledge/public/itc.aspx

[2] Improving Safety at Level Crossings, Japanese Developments. Y. Hirao RTTI Paper delivered to UIC International Railway Research Board Meeting, Paris $21^{\text {st }}$ November 2005. http://www.uic.asso.fr/html/monde/irbb-112005/pages/introduction.html

[3] Japan Railway Electrical Engineering Association, an Introduction of Signal "Level Crossing Protection Device" for the Railroad Engineer (in Japanese).

[4] N. Miyaguchi, M. Teramoto, K. Kumasaka, R. Ishima, T. Yasumoto, Y. Konno, S. Kanbe, Y. Fukuta, The Papers of Technical Meeting on Transportation and Electric Railway, IEE Japan, TER-12-42, pp. 13-17, 2012 (in Japanese). 
[5] M. Teramoto, N. Miyaguchi, K. Kumasaka, R. Ishima, Y. Fukuta, The papers of the 49th Symposium on the Use of Cybernetics on the Railways, No. 611, 2012 (in Japanese).

[6] Japan Railway Electrical Engineering Association, Railway and Electrical Engineering, Vol. 10, No. 11, pp. 37-41, 1999. 11 (in Japanese). 